\title{
Digital Structures and Light Movements
}

\author{
Esther Rolinson \\ Rolinson Craig Ltd \\ Hastings, UK \\ info@estherrolinson.co.uk
}

\author{
Sean Clark \\ Institute of Creative Technologies \\ De Montfort University, UK \\ seanc@cuttlefish.com
}

\begin{abstract}
This paper discusses 'Drawing Structures and Light Movements'; an experimental artwork consisting of a computer screen showing digitally captured drawings, and an LED matrix showing the movement patterns and rhythms derived from them. It was developed as part of Rolinson and Clark's on-going research into techniques for translating Esther Rolinson's drawings into light works. It makes use of a custom digital drawing tool made to accurately capture Rolinson's markmaking processes and replay them in real-time. Analysis of this drawing data has made it possible to extract rhythms from the drawings to create light sequences on an LED matrix. Rhythms that are not always immediately apparent within the original mark making appear to offer insights in to the drawing process and reveal something deeper about the relationships between hand-made and digital processes of construction. Esther Rolinson's drawings have a relationship to computerconstructed images and drawing machine processes. However, although they use repetitive algorithmic-like processes they are nuanced with spontaneity and gesture. This artwork examines the relationship between the form, timing, and sequential appearance of marks. It analyses fine differences between apparently identical processes, aiming to map subtle systems and structures in the drawings. It reflects a curiosity about structures embedded in human actions and sensations.
\end{abstract}

Digital art. Custom drawing tools. Light installations. LED matrix. Algorithmic art.

\section{INTRODUCTION}

Drawing Structures and Light Movements is an experimental artwork consisting of a computer screen showing digitally captured drawings and an LED matrix showing the movement patterns and rhythms derived from them. It has been developed as part of Esther Rolinson and Sean Clark's ongoing research into techniques for translating Esther Rolinson's drawings into light works. It makes use of a customised digital drawing tool made to capture Rolinson's mark-making processes and replay them in real-time. The artwork embodies the form, timing and sequential appearance of the marks, and reveals the interrelationships between them. It reflects the artist's curiosity about structures embedded in human actions and sensations. The aim of the work is to develop computational models of the drawings. The intention is to deepening understanding of the drawings themselves use these computational versions of the drawings as time based light works that seen on LED grids and inside three dimensional light structures. A first possible outcome of the project will be a computer program that can be used to analyse drawings and develop programming behaviours from them.

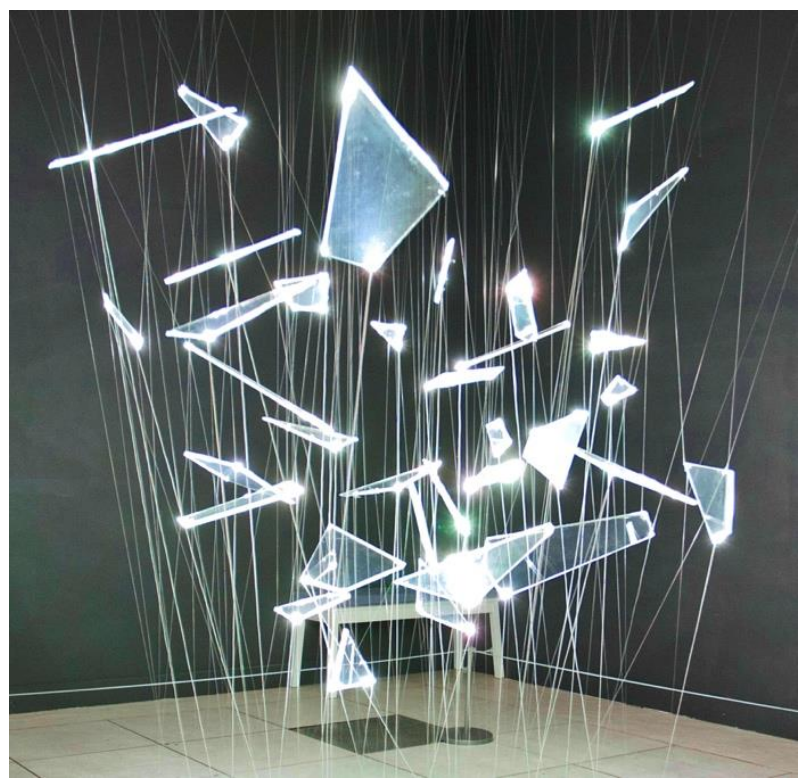

Figure 1: 'Splinter' Acrylic and programmed LED Light Installation by Esther Rolinson. 


\section{ABOUT THE ARTISTS}

Esther Rolinson makes light installations and sculptural works through a drawing process. The works are sensory representations of felt experience, memories and expressions of transitional states. The process of making them is meditative and the use of repeated actions and rules of construction allows for the artist's observation of thought connection to sensations.

Rolinson extends the drawing process into three dimensions, modelling forms and applying the same principles to construct large-scale works. These installation works are structural systems that are extendable kits of parts that can be assembled in many different ways in response to their site. At times, they are connected to environmental factors via the Internet such as temperature, light and humidity tying them into immediate states and subtle influences in their environment.

An on-going ambition in Rolinson's practice is to extend the flow of movement and form found in two and three-dimensional work into evolving time based sequences for lights. In this way, her sculptural installation systems are derived from instinctive human sensations found inside the drawing process.

Sean Clark's practice as an artist and programmer involves the creation of screen-based and 3D digital artworks that explore the nature of interactivity, flow and connectedness. Inspired by systems theory and cybernetics, he makes use of a wide range of computer hardware and software in his work, as well as 3D printing, electronics and digital lighting.

He has had a long interest in capturing and representing drawing activity that started in the early 1990s with his development of the ROCOCO Sketchpad multi-user drawing tool and drawing analyser (Scrivener \& Clark 1994; Clark and Scrivener 1993). He also developed a locative iPhone drawing app called Dropsketch that enabled user sketches to be tagged with their location and shared through a map-based interface (Clark 2013).

Clark has worked with Esther Rolinson on a number of her light installations, including Melt, Splinter and Thread, the award-winning installation Flown (Rolinson \& Clark 2016) that received the inaugural ArtCHI Exhibition Award First Prize and the Lumen Digital Art Prize Sculpture and 3D Award, both awarded in 2016 .

Rolinson and Clark's shared interest is in the connection between physical entities such as artefacts, virtual entities and systems and experiences through engagement with art. Their joint focus is on the use of complex but definable systems of construction.

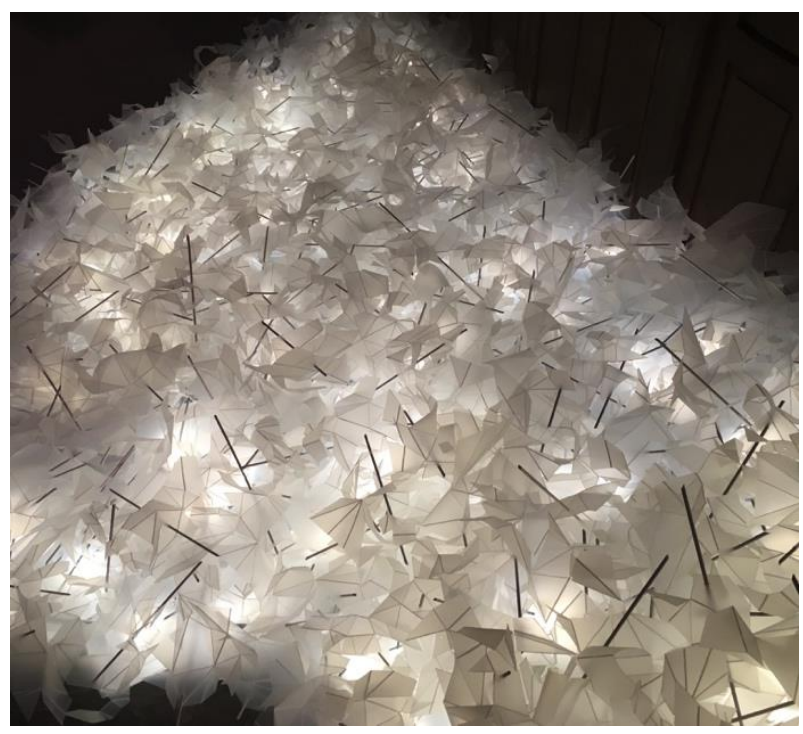

Figure 2: 'Flown' Hand-folded acrylic installation by Esther Rolinson developed in collaboration with Sean Clark.

\section{THE DRAWING PROCESS}

Esther Rolinson creates drawings in many ways, from simple pencil sketches to working in collaboration to create drawings through computer code. She approaches drawing as a spontaneous activity that could be likened to free-form dance in which rules and technique are learned but the outcome of 'doing it' cannot be predicted or completely controlled.

She uses drawing as process of 'thinking' to understand forms and sensation working with both instinctive and more studied approaches. As she develops an installation she makes sketches and detailed works. Each additional drawing adds another aspect of information and understanding to the emerging overall construction. This includes practical construction specifications and materials as well as expressions of intention. A set of drawings may at first be produced in two dimensions and then evolved as three-dimensional forms and as a rhythmic structure, but all the versions grow from the same underlying principles.

Rolinson's drawings have a strong relationship to computer-constructed images and drawing machine processes in that they employ repeated actions to build up forms in an algorithmic-like process. However, they are nuanced with spontaneity and gesture and each mark is unique rather than a true repetition. Like living things, they change and grow unpredictably. In using repeated gestures that produce ever changing marks there is 
an obvious humanness in the results. It might remind us of the impossibility of precise replication in the physical world and the continuous driving creativity of the organic world.

\section{DRAWING CAPTURE}

When specifying the new drawing capture software and related tools the goal was to investigate the extension of Esther Rolinson drawings into light sequences by looking for patterns and structures in her drawings that can be developed into programming behaviours.

This suggested that the development of a minimal drawing tool that focused on capturing the timing and precise movements in Rolinson's mark making. It should not interfere with the natural drawing process. As with the ROCOCO Sketchpad (ibid) it was felt that any excess user interface would be a distraction to the user, and any temptation to add multiple pen types, rulers and similar features was resisted.

A simple mark-making tool was created consisting of a single 'pen' and, after some discussion, a colour palette and pen-thickness selector. An 'undo' feature was added to enable erroneous marks to be removed, although it was agreed that its use should be limited to the removal of glitches, rather than as a part of the drawing process itself.

The tool is optimised for use on an iPad or similar tablet, although it will work on a mobile phone or desktop computer. It captures all of the events related to the mark-making process, including the start, movement and end of each mark, the selection of colour and pen sizes and any undo's. Clearly this is a vast simplification of the full subtleties of the mark-making process, but prior experience suggested that it would be a suitable starting point for the work.

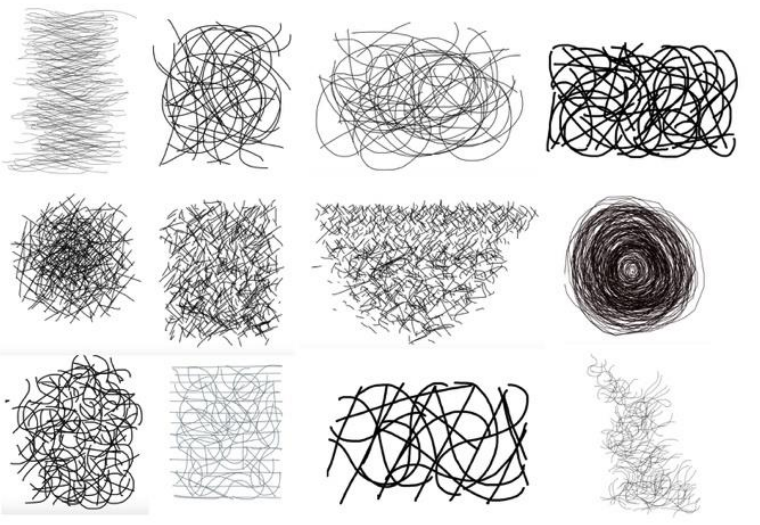

Figure 3: Drawings created using the digital drawing tool.
Drawings made using the tool can be saved and played back in real-time. Every mark-making event captured is time-stamped so that both the rhythms within and between marks can be extracted.

In the first use of the tool about fifty drawings have been made. Analysis of this drawing data has so far focused on extracting the time taken to make a mark and the delays between mark-making.

\section{RE-PRESENTING DRAWINGS AS LIGHT}

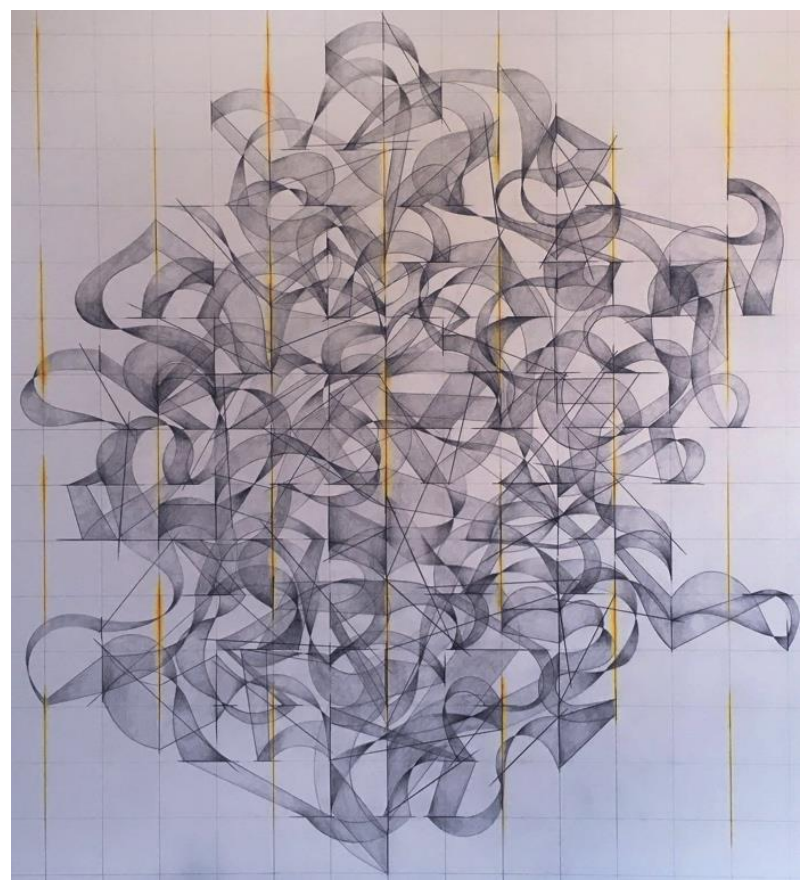

Figure 4: Revolve drawing by Esther Rolinson.

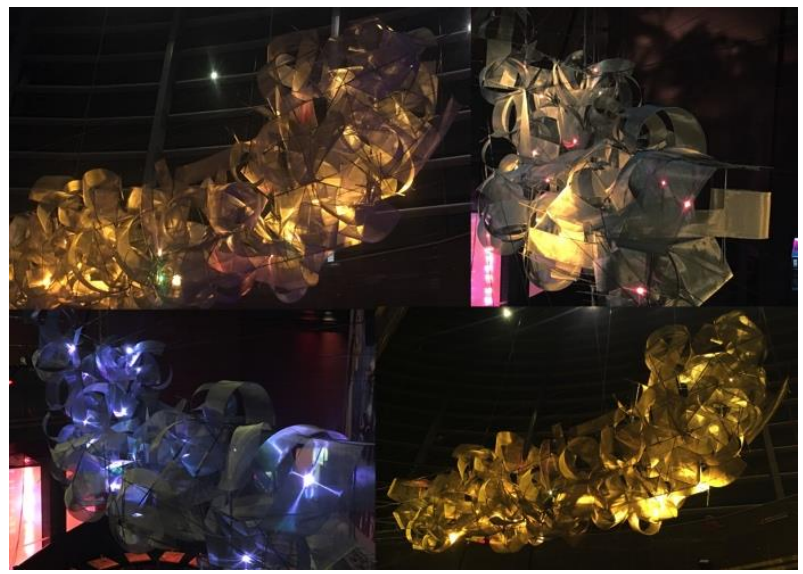

Figure 5: 'Revolve' Light Installation by Esther Rolinson programming developed in collaboration with Sean Clark.

The use of this data in new artworks began with the installation artwork Revolve, built as a temporary work for the Inside Out Festival at Curve Theatre, Leicester in autumn 2017. In this installation, Rolinson began with a pencil drawing consisting of a set of rules rectangles arranged in a pattern of a 
straight, a curve and a twist. This was then used as the basis for a large-scale artwork constructed using a metallic mesh interspersed with LED lamps. The LEDs were then programmed by Sean Clark to change brightness in accordance with the rhythms found within the construction data for the original Revolve drawing.

For the experimental artwork Digital Structures and Light Movement, Rolinson and Clark have taken a different approach, playing the data live on A4 sized screens that show them developing in real time. They have also included an LED matrix showing the rhythms of the drawings presented as light as well as a demonstration of the drawing capture tool paired with a simple drawing machine that re-creates the digital drawings in physical form. This set of re-presentations of the drawing data is intended to demonstrate to the viewer the range of creative possibilities being exploring within the work.

\section{DISCUSSION}

Both Revolve and Digital Structures and Light Movement raise questions that require many experiments to possibly answer. The connections between the drawing process and digital practice are becoming more evident, but the current representations of the drawing data do not yet convey the structures or complexity of the patterns in the drawings.

In Revolve, the light rhythms, while intriguing to both the artists and the viewers, are a preliminary experiment that so far has served to map out the next stages of work.

The questions raised by the work include:

- How can a structure be recognized in a drawing?

- How can a drawing structure be mapped as a time sequence?

- How can a drawing structure be re-drawn by a computer?

- How can overall patterns of forms or timings be recognized?

- How can the timings be translated into light sequences in a meaningful way?

- How can a curve be expressed as a light sequence?

- What generates speeds and changes in light levels?

To explore solutions, Rolinson and Clark are carrying out further creative experiments. These include identifying both straight-forward connections between drawing and lighting sequences and more abstract comparisons and rearrangements of the drawing material that may assist in distinguishing patterns in their construction.

A direct method has been to connect the LED matrix to the drawing capture tool so that it mimics the marks as they are made, fading up and down with varying degrees of pressure. This is an effective and a fun tool that informs the process but is not an end point in itself for this work. Another aim is to use information from the app to generate marks as a response or 'answer' to Esther Rolinson's marks, in effect, creating a feedback loop between computer and hand drawing. To do this we have been using an Arduino powered drawing machine to mimic and respond to human mark-making.

A practical and conceptual point of enquiry is around the use of repetition. As described earlier similar marks made in a hand drawing appear to be repetitive, but one mark cannot be a true copy of another. The computer is able to make exact replications on screen and draw marks that are accurate to the eye when operating a drawing machine. As this line of work is developed, Rolinson and the computer will produce multiple marks using the same principles of construction. These will be compared with each other to see if the differences between the two reveal additional patterns that may assist in developing light-based interpretations of the drawing.

\section{CONCLUSIONS}

The overall ambition of the project is to further understanding of Esther Rolinson's drawing structures and translate the drawings into computer-controlled lighting behaviours that can be shown in two and three-dimensional light works. To enable this to happen the first development stage is to write a computer program that can be used to analyse drawings and develop programming behaviours from them.

However, the process of studying the patterns and rhythms not always immediately apparent within the original artworks generates other interesting areas of work. It appears to offer insights into the relationships between hand-made and digital processes of construction. It has the potential to create a subtle exchange between human and computer in which both may be able to incorporate actions from the other. 


\section{REFERENCES}

Scrivener, S. and Clark, S. (1994) Sketching in Collaborative Design. Interacting with Virtual Environments. Wiley Professional Computing, Editors: Lindsay MacDonald, John Vince.

Clark, S. and Scrivener, S. (1993) Using Computers to Capture and Structure Drawing
Surface Activity. The First Symposium on Creativity and Cognition. Loughborough, UK.

Clark, S. (2013). Dropsketch Installation. Proceedings of the $9^{\text {th }}$ ACM Conference on Creativity and Cognition. Sydney, NSW, Australia.

Rolinson, E. and Clark, S (2016) 'Flown' Sculptural Light Installation. CHI'2016, San Jose, CA, USA. 